Supporting Information

\title{
GSH-responsive drug delivery system for active therapy and reducing side effects of bleomycin
}

Man Zhang, ${ }^{\dagger}$ Chao Jia, ${ }^{*}$ Ji Zhuang, ${ }^{*}$ Yuan-Yuan Hou, ${ }^{\dagger}$ Xi-Wen He, ${ }^{*}$ Wen-You Li ${ }^{*}, *$, Gang Bai ${ }^{*}+$, Yu-Kui

Zhang ${ }^{*}, \bar{t}, s$

$†$ College of Pharmacy, Nankai University, Tianjin 300071, China.

\$ State Key Laboratory of Medicinal Chemical Biology, Tianjin Key Laboratory of Biosensing and Molecular Recognition, Research Center for Analytical Sciences, College of Chemistry, Nankai University, Tianjin 300071, China.

§National Chromatographic Research and Analysis Center, Dalian Institute of Chemical Physics, Chinese Academy of Sciences, Dalian 116023, China.

\section{Corresponding Author}

*E-mail: wyli@nankai.edu.cn.

*E-mail: gangbai@nankai.edu.cn.

*E-mail: ykzhang@dicp.ac.cn 
Materials and Reagents: Tetraethyl orthosilicate (TEOS), 3-aminopropyl-triethoxysilane (APTES), 1-(3-dimethylaminopropyl)-3-ethylcarbodiimide hydrochloride (EDC) and cetyltrimethylammonium bromide (CTAB) were purchased from J\&K Technology Co., Ltd. (Beijing, China); $\mathrm{KMnO}_{4}$ and $\mathrm{Na}_{2} \mathrm{CO}_{3}$ were purchased from BoHua Chemical Reagent Co., Ltd. (Tianjin, China); N-hexane, n-hexanol and triton X-100 were purchased from Heowns Biochemical Technology Co., Ltd. (Tianjin, China). Polyacrylamine hydrochloride (PAH); $\mathrm{NH}_{2}-$ $\mathrm{PEG}_{2000}-\mathrm{NH}_{2}$ and Calcein-AM/PI double stain kit were purchased from Solarbio Biotechnology Co., Ltd. (Shanghai, China). The water used in the experiment was ultrapure water $(18.25 \mathrm{M} \Omega \bullet \mathrm{cm})$. 4T1 mouse breast cancer cell (4T1 cell), human bronchial smooth muscle cells (HBSMC cells) were purchased from Enzyme Research Biotechnology Co., Ltd. (Shanghai, China). RPMI 1640 cell culture medium and Dulbecco's modified eagle medium (DMEM) culture medium was purchased from ThermoFisher Scientific Co., Ltd. (USA).

Instruments Information: The ultraviolet-visible absorption spectrum was measured by UV2450 ultraviolet-visible spectrophotometer (Shimadzu, Japan). The TEM photograph was obtained by HT7700 Exalens microscope (Hitachi, Japan), and the HRTEM image was obtained by JEM2800 (JEOL, Japan). Scanning electron microscope (SEM) photograph and X-ray energy spectrum analysis (EDS) were measured by Apreo S LoVac (EDAX, America), Fourier transform infrared spectroscopy (FTIR, 4000-400 $\mathrm{cm}^{-1}$ ) was measured by a TENSOR II Fourier Transform Infrared Spectrometer (Bruker, Germany). MRI images was obtained by HT-MRSI60-35A 1.5T MRI instrument (Shinning, China). The lung function of mice was measured by AniRes 2005 animal lung function analysis system (Beijing beilanbo Technology Co., Ltd, Beijing, China). 


\section{Preparation of hollow mesoporous silica nanoparticles modified by PEG (HMSN@PEG):}

Silica nanoparticles $(50 \mathrm{mg})$ was added into $10 \mathrm{~mL}$ of $\mathrm{H}_{2} \mathrm{O}$ for 15 minutes, and then the mixed system was added into $30 \mathrm{~mL}$ of ethanol: water $=1: 1$ solution containing $75 \mathrm{mg}$ of CTAB and $0.275 \mathrm{~mL}$ of $\mathrm{NH}_{3} \cdot \mathrm{H}_{2} \mathrm{O}$, and stirred at room temperature for 45 minutes. Next TEOS $(0.125 \mathrm{~mL})$ was added slowly, and continue to stir for 3 hours. The products were collected by centrifugation and washed with $\mathrm{H}_{2} \mathrm{O}$ and ethanol for three times. The collected product was redispersed in $10 \mathrm{~mL}$ of $\mathrm{H}_{2} \mathrm{O}$ contained $\mathrm{Na}_{2} \mathrm{CO}_{3}(212 \mathrm{mg})$. $\mathrm{HMSN}$ was obtained by stirring for $15 \mathrm{~h}$ under $50{ }^{\circ} \mathrm{C}$, and centrifuging and washing with $\mathrm{H}_{2} \mathrm{O}$ for 3 times.

Finally, $50 \mathrm{mg}$ HMSN were dispersed in water $(50 \mathrm{~mL}), 200 \mathrm{mg}$ PAH was added to them, ultrasonic wave was applied for $2 \mathrm{~h}$, and then centrifuged and washed for 3 times to obtain the product HMSN@PAH, which was dispersed in ultrapure water $(8 \mathrm{~mL})$ for use. $\mathrm{NH}_{2}-\mathrm{PEG}_{2000}-\mathrm{NH}_{2}$ (200 mg) and EDC (40 mg) were dissolved in ultrapure water (3 mL) and stirred for $30 \mathrm{~min}$, and then $2 \mathrm{~mL}$ of activated solution was added to the solution which contained HMSN@PAH. The reaction time was $12 \mathrm{~h}$. The product HMSN@PEG was obtained by centrifuging and washing with ultrapure water for three times.

Calein-AM/PI Double Staining Experiment: 4T1 cells were seeded in 6-well plates. When the growth density reached $80 \%$ of the maximum density, the culture medium was removed, and the RPMI 1640 culture medium, $64 \mu \mathrm{g} / \mathrm{mL}^{\mathrm{HMnO}} \mathrm{HMnO}_{2} @ \mathrm{PEG}, \mathrm{HMnO}_{2} @ \mathrm{PEG} / \mathrm{BLM}$ and loading equivalent free BLM was added respectively. After incubation for $12 \mathrm{~h}$, the current culture medium was removed and the cells were stained with calein-AM/PI double staining kit. The excitation/emission wavelength of calein am was $490 \mathrm{~nm} / 515 \mathrm{~nm}$, and the excitation/emission 
wavelength of PI was $488 \mathrm{~nm} / 617 \mathrm{~nm}$.

As a contrast, $\mathrm{HMnO}_{2}, \mathrm{HMnO}_{2} @ \mathrm{PEG}, \mathrm{HMnO}_{2} @ \mathrm{PEG} / \mathrm{BLM}$ was used under the same conditions, the double staining procedure was repeated with HBSMC cells except that the culture medium was changed to DMEM.

Establishment of Tumor Model: 4T1 cells $\left(3 \times 10^{6}\right)$ were injected subcutaneously to the left inferior temporal region of BALB/c nude mice and the tumor model was established. The treatment experiment of the model nude mice was started after the tumor grew to a size of $40-100 \mathrm{~mm}^{3}$. Tumor volume was calculated by the formula: tumor volume $=\left[\right.$ length $\left.\times(\text { width })^{2}\right] / 2$. The length and width of each tumor were determined using a vernier caliper.

In Vivo Toxicity Test of $\mathrm{HMnO}_{2} @$ PEG: After the research of anti-tumor in vivo and pulmonary function test, the internal organs (heart, liver, spleen, lung, kidney) of the BALB/c nude mice from control group, free BLM group, $\mathrm{HMnO}_{2} @ \mathrm{PEG}$ group or $\mathrm{HMnO}_{2} @ \mathrm{PEG} / \mathrm{BLM}$ group were removed and stored in paraformaldehyde (4\%) for histological analysis by hematoxylin and eosin (HE) staining and masson staining to evaluate the toxicity of each group. 


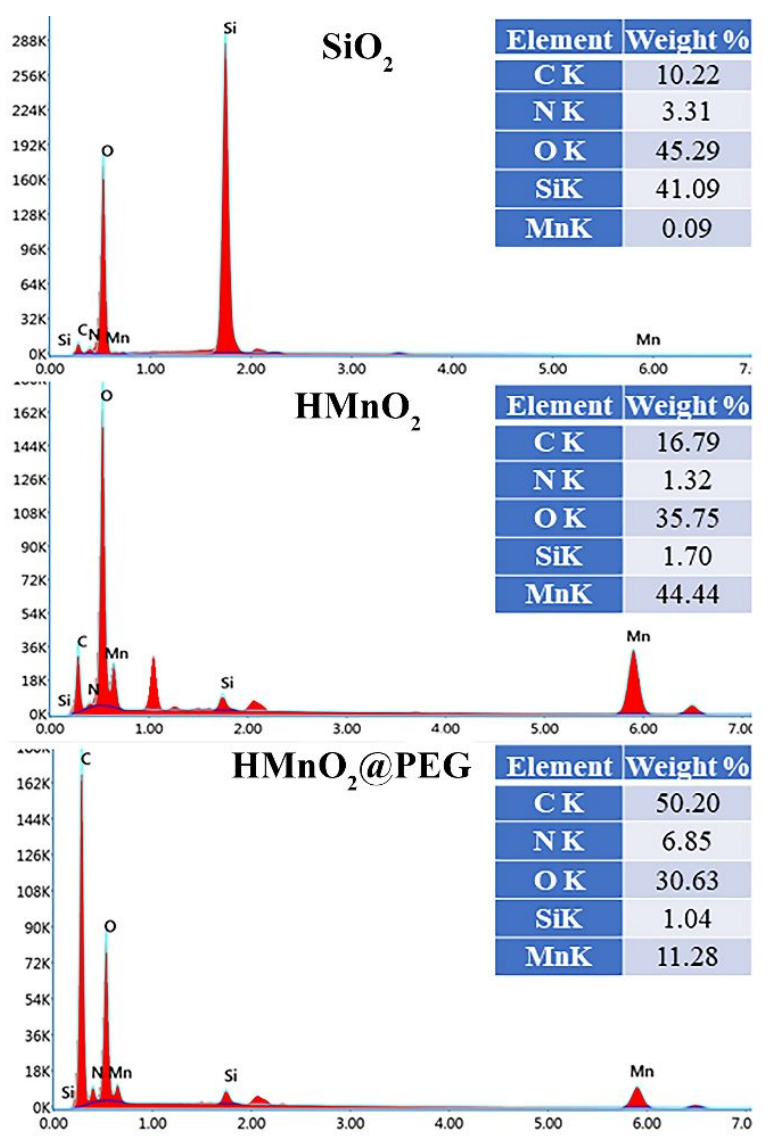

Figure S1. EDS analysis of $\mathrm{SiO}_{2}, \mathrm{HMnO}_{2}$ and $\mathrm{HMnO}_{2} @$ PEG.

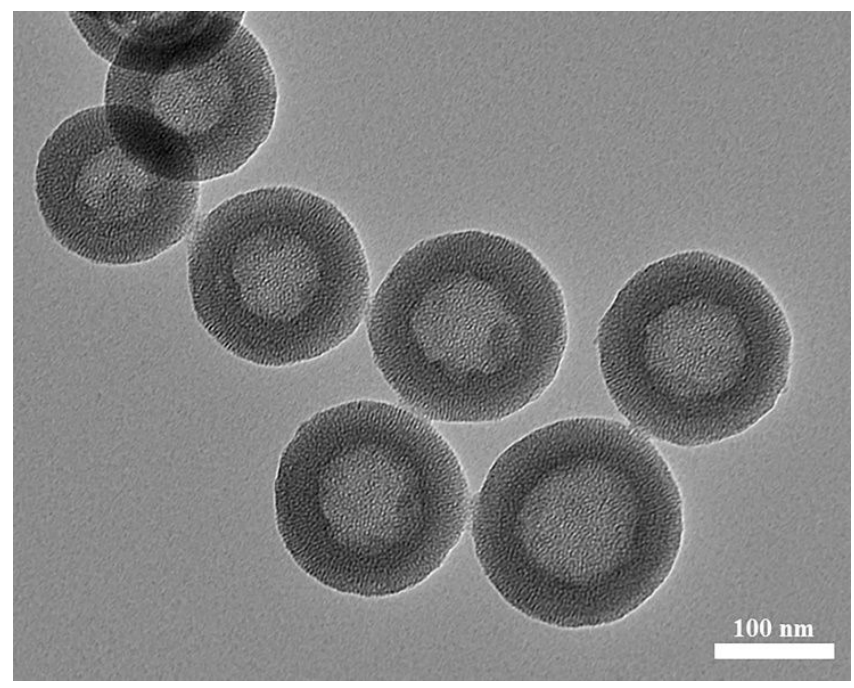

Figure S2. TEM photo of HMSN@PEG 


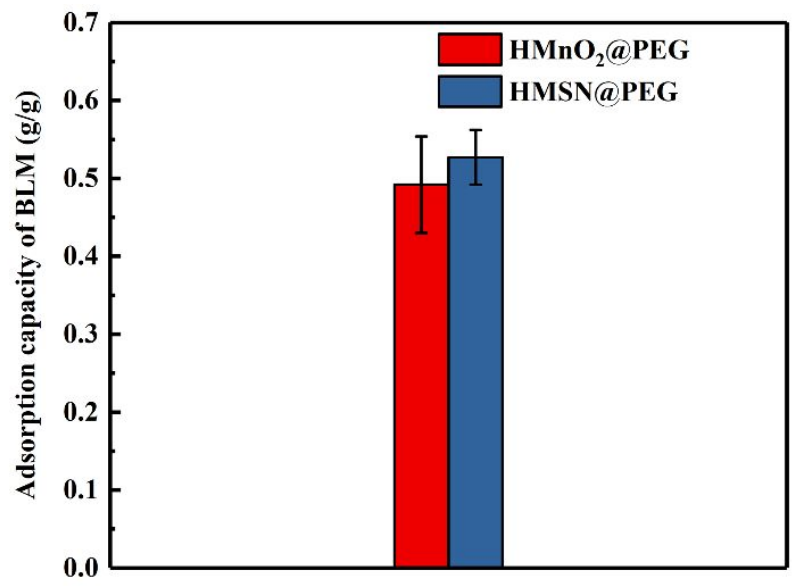

Figure S3. Adsorption capacity of BLM by HMnO2@PEG or HMSN@PEG. The data show mean \pm SEM, representative from 3 independent experiments. 

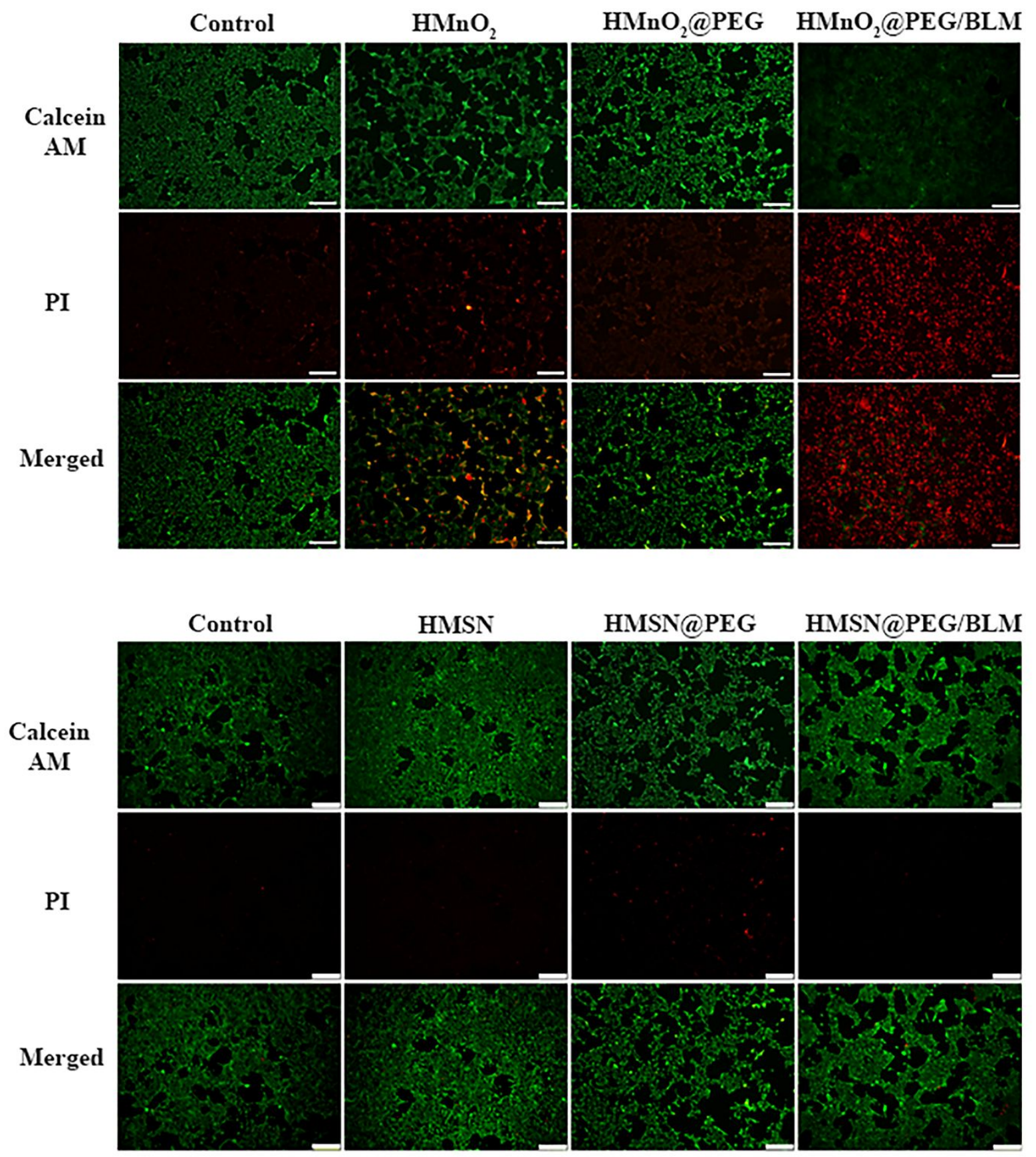

Figure S4. Calcein-AM/PI staining and merged photos of 4T1 cells incubated by $\mathrm{HMnO}_{2}, \mathrm{HMnO}_{2} @ \mathrm{PEG}$, $\mathrm{HMnO}_{2} @$ PEG/BLM and free BLM, HMSN@PEG, HMSN@PEG/BLM. The scale bar in the photos was 20 $\mu \mathrm{m}$. 
(a)
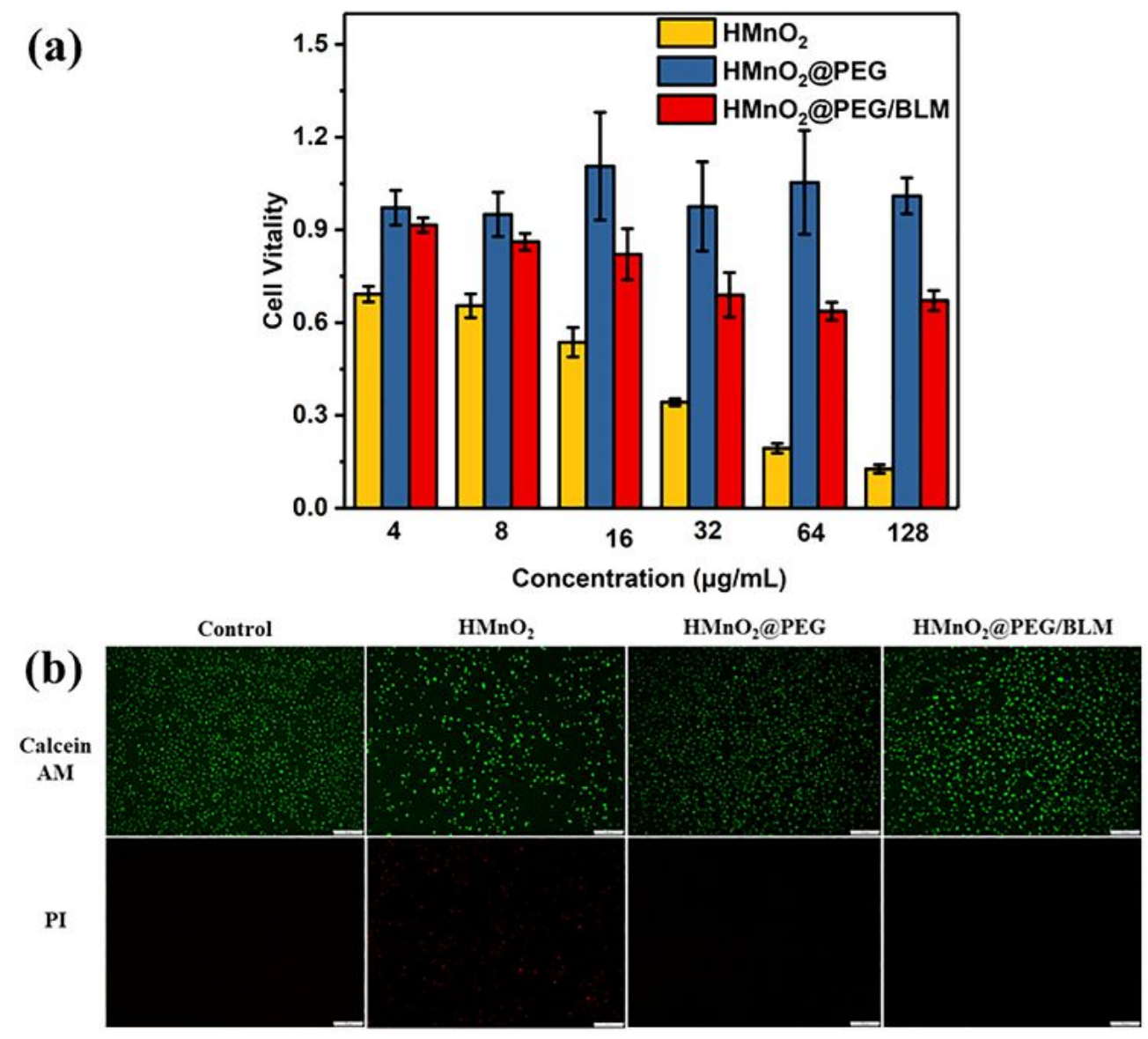

Figure S5. (a) $\mathrm{HBSMC}$ cytotoxicity test of $\mathrm{HMnO}_{2}, \mathrm{HMnO}_{2} @ \mathrm{PEG}, \mathrm{HMnO}_{2} @ \mathrm{PEG} / \mathrm{BLM}$; (b) Calcein-AM/PI staining of $\mathrm{HBSMC}$ cells incubated by $\mathrm{HMnO}_{2}, \mathrm{HMnO}_{2} @ \mathrm{PEG}, \mathrm{HMnO}_{2} @ \mathrm{PEG} / \mathrm{BLM}$. The scale bar in the photos was $20 \mu \mathrm{m}$. The data show mean $\pm \mathrm{SD}(\mathrm{n}=3)$. 

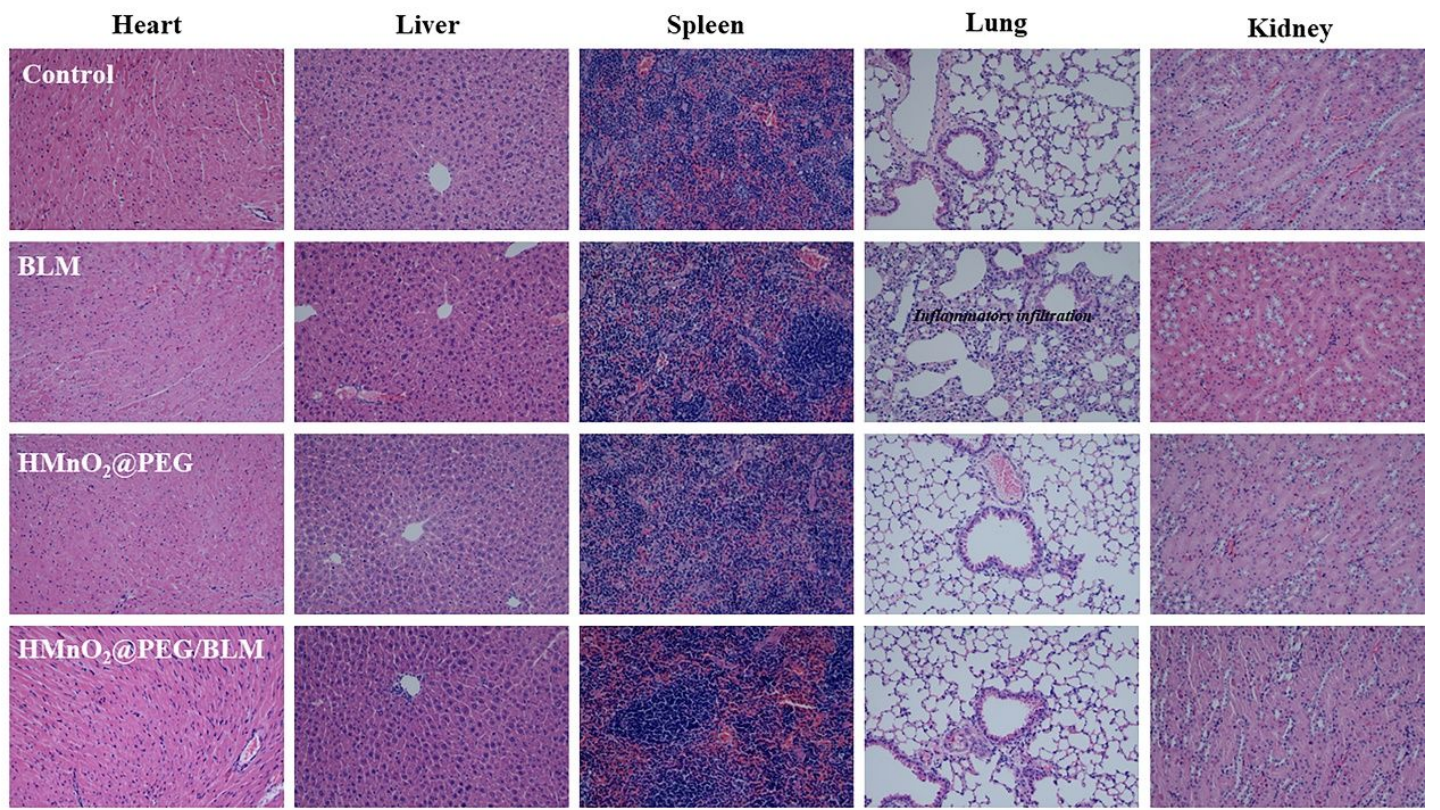

Figure S6. HE staining photos of main organs of mice in each group after treatment. 